\section{Toll like receptor 4 (TLR4) expression on peripheral blood mononuclear cells (PBMCs) among patients with Graves disease}

\author{
Youssra G. Mahfouz ${ }^{1}$, Salwa S. Hosny ${ }^{2}$, Mohamed \\ N. Farres ${ }^{1}$, Dina S. Sheha ${ }^{1}$, Dina A. Marawan ${ }^{2}$ and \\ Hossam M. ElKady ${ }^{1}$
}

\author{
${ }^{1}$ Department of Internal Medicine, Allergy and Clinical \\ Immunology Unit, Faculty of Medicine, Ain Shams University, \\ Cairo, Egypt. \\ ${ }^{2}$ Department of Internal Medicine, Endocrinology Unit, Faculty \\ of Medicine, Ain-Shams University, Cairo, Egypt.
}

The Egyptian Journal of Immunology Volume 28 (4), 2021: 215-223. www.Ejimmunology.org

\begin{abstract}
Graves disease (GD) is a multifactorial disease due to multiple environmental and genetic factors as well as immune malfunction. Human Toll-like receptors (TLRs) play a key role in activating innate and adaptive immune cells. Their role in modulating immunity also interferes with the mechanisms that maintain tolerance in the host. Thus, expression or activation of TLRs can contribute to the loss of tolerance by a lot of mechanisms. In order to confirm the importance of TLR4 in the pathogenesis of GD, this study intended to measure TLR4 expression on peripheral blood mononuclear cells in GD patients before and after control of disease with Carbimazole as compared to a group of normal controls. We conducted a case-control study on 36 patients with newly diagnosed Graves disease and 36 individuals as the control group. Patients were recruited from the endocrinology outpatient clinic, Ain-Shams University Hospitals and were followed up till achieving the euthyroid state (for a minimum period of 6 months). In GD patients at baseline, the mean monocyte percentage was $4.46 \%$, and the mean TLR4 on monocytes $90.91 \%$. After achieving euthyroid state, the mean monocyte was $6.16 \%$, and the mean TLR4 on monocytes $72.30 \%$. In the control group, the mean monocyte was $3.88 \%$, and the mean TLR4 on monocytes $66.30 \%$. Results indicated significant differences in expression of TLR4 between GD patients before treatment, after achieving euthyroid state and in the control group $(p<0.0001)$. In conclusion, the present study showed a higher expression of TLR4 on monocytes among newly diagnosed GD patients in comparison to normal individuals. TLR4 expression on monocytes decreased significantly among GD patients after treatment.
\end{abstract}

Keywords: TLR4, Graves disease

Date received: 29 July 2021; accepted: 22 September 2021 


\section{Introduction}

Graves disease (GD) is a prototypical organspecific autoimmune disease. The etiology is multifactorial; immune tolerance dysregulation occurs as a result of interactions between environmental and genetic predisposing factors. ${ }^{1}$ Autoantibodies present in GD are responsible for binding and activating the thyroid stimulating hormone receptor, causing hyperthyroidism as well as goiter. ${ }^{2}$

Previous studies have demonstrated the significance of aberrant activation of the innate immune response on the pathogenesis of the disease. Innate immune system recognizes a wide range of pathogens by a number of receptors called pattern recognition receptors (PRRs). Human Toll-like receptors (TLRs), which are part of PRRs were the first receptors identified to sense the presence of microorganisms and mount innate immune responses. ${ }^{3}$ They are expressed on all innate immune cells, including macrophage, neutrophils, natural killer (NK) cells, dendritic cells (DCs), mast cells, basophils, and eosinophils. ${ }^{4}$

Activation of TLRs stimulate signaling cascades as a defense mechanism towards invaders and to repair injured tissue, leading to the release of various immune modulators and inflammatory cytokines. ${ }^{4}$ Excessive TLR activation cause sustained release of proinflammatory cytokines and chemokine production which may disrupt the immune homeostasis and, as a result, leads to the progress and development of several autoimmune diseases. ${ }^{4}$

TLR4 has been shown to identify the bacterial endotoxin lipopolysaccharide (LPS), which is an integral part of all gram-negative bacteria's outer surface. Bacterial endotoxin LPS may play key role in modifying thyroid-specific gene expression by up-regulating TSHstimulated thyroglobulin. ${ }^{5}$

In addition to infection, ischemia/reperfusion damage, trauma, or other noxious stimuli could all cause sterile tissue damage. Tissue damage causes innate immune responses to repair the damage by releasing intracellular molecules and/or cleaving extracellular matrix molecules. TLR4 recognizes such molecules, which are now known as danger (or damage)-associated molecular patterns (DAMPs). ${ }^{6}$ Therefore, innate immune activation plays essential roles in the death and clearance of pathogens and dead cells, as well as in producing local inflammation and triggering acquired immunological responses. Those reactions may be directed against autoantigens in the thyroid. ${ }^{6}$

Consequently, in order to confirm the importance of TLR4 in the pathogenesis of GD, the present study was conducted to measure TLR4 expression on peripheral blood mononuclear cells in patients with GD before and after treatment of the disease with Carbimazole as compared to a group of normal controls

\section{Subjects and Methods}

This case control study included 36 patients with newly diagnosed Graves disease with and without ophthalmopathy. Patients were recruited from the endocrinology outpatient clinic, Ain-Shams University Hospitals from November 2018 till October 2020.

-Graves disease was diagnosed according to the American thyroid association guidelines. ${ }^{7}$

-Patients with any chronic or other endocrinology disease and subjects receiving corticosteroids or any immunosuppressive drugs were excluded from our study. The study also included 36 apparently healthy individuals, forming a control group matched with patients for age and sex.

-The study protocol was reviewed and approved by the Ethical Committee of the Faculty of Medicine, Ain Shams University (Approval No. FMASU MD 265/2018). Accordingly, a written informed consent was obtained from each participant included in our study.

\section{Methods}

All patients were subjected to full medical history taking with emphasis on hyperthyroid symptoms and general examination including BMI evaluation, pulse, skin and hand 
examination, Cardiovascular, Neurological and Neck assessment.

\section{Assessment of severity of exophthalmos}

This was performed using EUGOGO Classification of the Severity of the Ophthalmopathy. ${ }^{8}$ The disease was classified as mild, moderate, severe, or sight-threatening as follows: Mild cases usually presented with one or more signs including minor retraction of the lid $(<2 \mathrm{~mm})$, mild involvement of soft tissue, exophthalmos $<3 \mathrm{~mm}$, absent or transient diplopia and exposure of cornea with response to lubricants. Moderate to severe patients without sight-threatening Graves ophthalmopathy whose eye disease has sufficient impact on daily life to justify the risks of immunosuppression (if active) or surgical intervention (if inactive). Patients usually presented with one or more signs including retraction of the lid $(>2 \mathrm{~mm})$, moderate or severe involvement of soft tissue, exophthalmos $\geq 3 \mathrm{~mm}$ and constant diplopia. Sight-threatening Graves ophthalmopathy patients usually presented with severe exposure of the eye leading to dysthyroid optic neuropathy or corneal breakdown. Other infrequent cases are ocular globe subluxation, severe forms of frozen eye, choroidal folds, and postural visual darkening's. This category warrants immediate intervention.

\section{Laboratory investigations}

These were performed for patients with GD at baseline and after treatment with carbimazole for a minimum period of 6 months. The same laboratory investigations were preformed once to the control group.

Laboratory investigations included Toll like receptor 4 (TLR 4) expression on peripheral blood mononuclear cells (PBMCs) by flowcytometry (Figure 1, 2).

\section{TLR4 expression by flowcytometry}

A total of $2 \mathrm{ml}$ of a fresh venous blood sample on EDTA were used for determination of TLR4 expression on PBMCs. Plastic citrated tubes were used for blood collection. A total of $50 \mathrm{ml}$ of EDTA-anticoagulated whole blood were added to each tube, fluorochrome conjugated anti-TLR4 antibodies with determined appropriate volume were added in the tubes, then lysed using $1 \mathrm{ml}$ lysing solution and left in the dark and room temperature for 2-10 minutes till complete RBCs lysis and the suspension became clear. Data acquisition and analysis were performed on Navios flow cytometer (Coulter, Electronics, Hialeah, FL, USA) according to the manufacturer's instruction. PBMCs were gated according to their forward and side scatter properties, and TLR4 expressed cells were gated out of total PBMCs. TLR4 expression was determined as a percentage of gated TLR4 positive PBMCs.

\section{Statistical methods}

IBM@ SPSS@ Statistics version 23 (IBM@ Corp., Armonk, NY) was used to analyze the data. The Pearson chi-squared test was used to compare intergroup differences, and categorical variables are represented as ratios, numbers, and percentages. Normally distributed numerical data were presented as mean and standard deviation and between-group differences were compared using the independent-samples $\mathrm{t}$ test. The paired-samples t-test was used to compare paired numerical data showing a normal distribution. Skewed numerical data were presented as median and interquartile range and between-group differences were compared with the Mann-Whitney test. Paired numerical data showing non-normal distribution were compared with the Wilcoxon signed ranks test. Receiver-operating characteristics (ROC) curve analysis was used to examine the diagnostic value of TLR4. The area under the ROC curve (AUC) is interpreted as follows: $<0.6$ = fail, $0.6-0.69=$ poor, $0.7-0.79=$ fair, $0.8-$ $0.89=$ good, $\geq 0.9=$ excellent. The DeLong method was used to compare area under different ROC curves. The Bonferroni method was used to adjust the level of significance for subgroup analysis. Two-sided $P$-values $<0.05$ were considered statistically significant. 


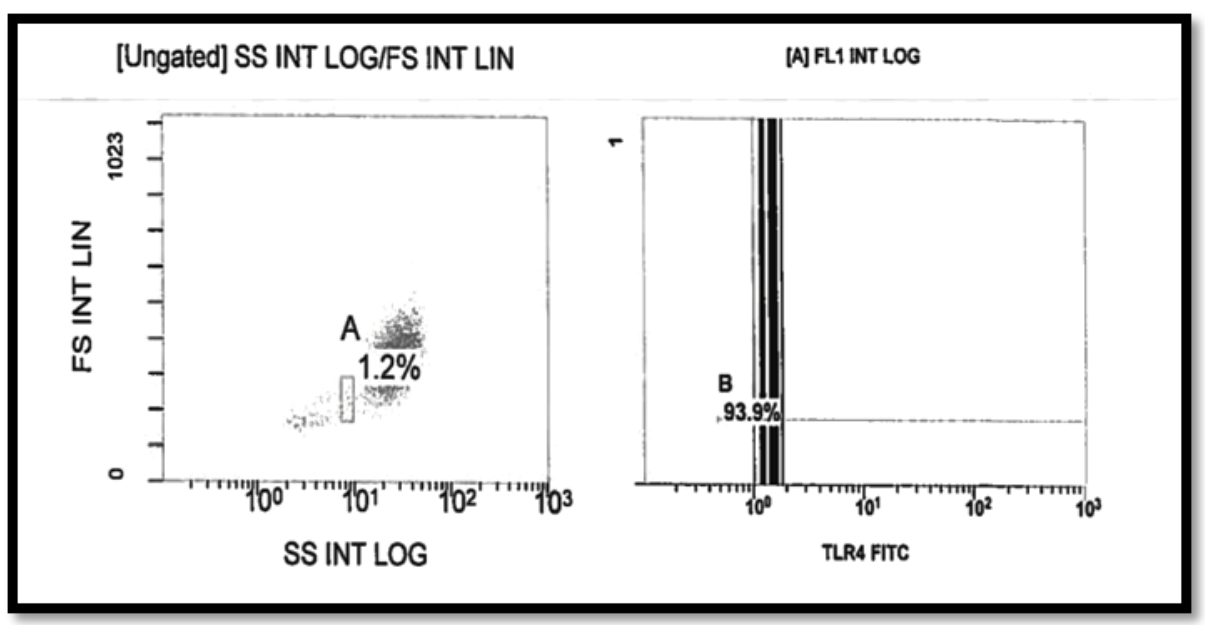

Figure 1. Characterization and gating of TLR4 in one of the cases before treatment.

Figure (A) shows the percentage of gated monocytes. Figure (B) shows the percentage of TLR4 expression on gated monocytes.

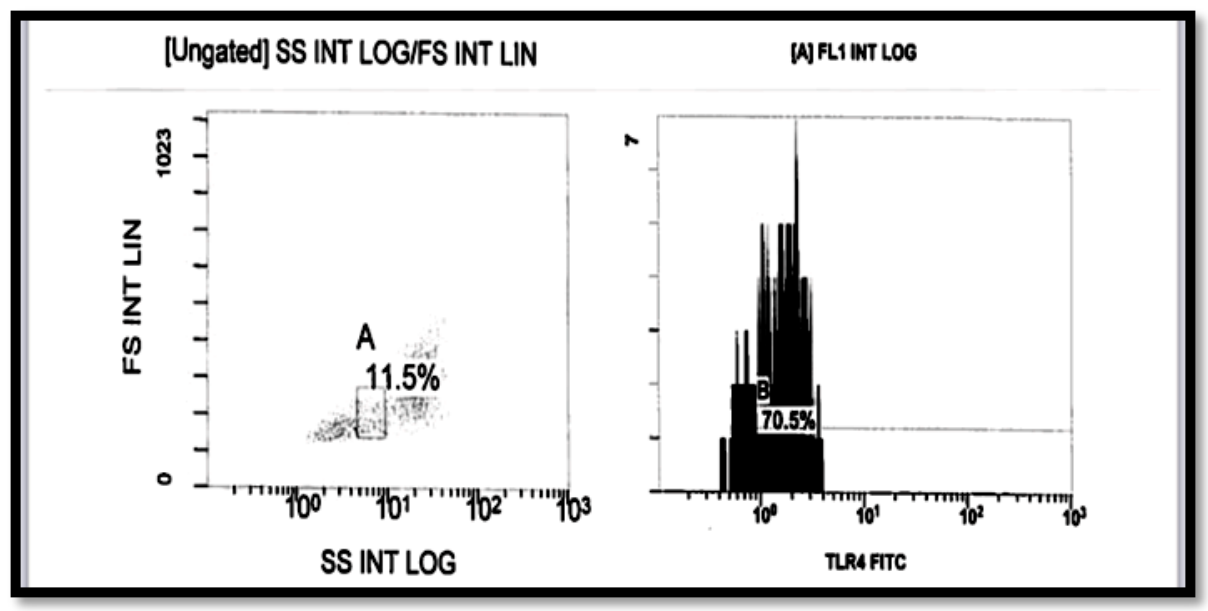

Figure 2. Characterization and gating of TLR4 in the same case after treatment.

Figure (A) shows the percentage of gated monocytes. Figure (B) shows the percentage of TLR4 expression on gated monocytes.

\section{Results}

A total of $36 \mathrm{GD}$ patients were included in this study (22 females, 14 males), their mean age was $28.5 \pm 6.9$ years and mean BMI $20.3 \pm 2.00$ $\left(\mathrm{kg} / \mathrm{m}^{2}\right)$. A total of 36 controls were included in this study (20 females, 16 males), their mean age was $28.5 \pm 5.5$ years and mean BMI $23.0 \pm$ $1.5\left(\mathrm{~kg} / \mathrm{m}^{2}\right)$. There was no significant difference between GD cases and controls regarding age $(P=0.98)$ or gender $(p=0.63)$. However, BMI was significantly higher among controls than $G D$ cases $(P<0.0001)$
Exophthalmos was observed among $20 \mathrm{GD}$ patients $(55.6 \%), 9$ patients had mild exophthalmos, whereas 11 showed moderate to severe exophthalmos. Treatment with Carbimazole ranged from 6 to 17 months, mean \pm SD was $11.1 \pm 2.9$ months.

TLR4 expression on monocytes was significantly higher among GD cases at baseline than among controls, whereas monocyte count did not differ significantly between both groups (Table 1). 
Table 1. Comparison of TLR4 on monocytes and monocyte count in cases before treatment versus controls

\begin{tabular}{|c|c|c|c|c|c|}
\hline \multirow[t]{2}{*}{ Variable } & $\begin{array}{l}\text { GD cases } \\
\text { before } \\
\text { treatment } \\
(n=36)\end{array}$ & $\begin{array}{c}\text { Controls } \\
(n=36)\end{array}$ & \multirow[t]{2}{*}{ Difference } & \multirow[t]{2}{*}{$95 \% \mathrm{Cl}$} & \multirow[t]{2}{*}{$\begin{array}{c}P- \\
\text { value }^{\S}\end{array}$} \\
\hline & Mean $\pm S D$ & Mean $\pm S D$ & & & \\
\hline TLR4 on monocytes (\%) & $90.91 \pm 5.06$ & $66.30 \pm 27.24$ & -24.62 & -33.82 to -15.41 & $<0.0001$ \\
\hline Monocyte count (\%) & $4.46 \pm 2.12$ & $3.88 \pm 2.13$ & -0.58 & -1.58 to 0.42 & NS \\
\hline
\end{tabular}

$\S$. Independent-samples t-test. $P>0.05$ is no significant (NS).

After treatment with Carbimazole, TLR4 reduced among GD patients, whereas monocyte expression on monocytes was significantly count increased significantly (Table 2).

Table 2. Comparison of TLR4 on monocytes and monocyte count in GD cases before and after treatment

\begin{tabular}{lcccccc} 
& & $\begin{array}{c}\text { GD patients } \\
\text { Before } \\
\text { treatment }\end{array}$ & $\begin{array}{c}\text { GD patients } \\
\text { After } \\
\text { treatment }\end{array}$ & & \multicolumn{3}{c}{ Paired differences } & \\
\hline Variable & $\mathrm{n}$ & Mean \pm SD & Mean \pm SD & Mean \pm SD & $95 \% \mathrm{Cl}$ & $P$-value \\
\hline $\begin{array}{l}\text { TLR4 on } \\
\text { monocytes (\%) }\end{array}$ & 36 & $90.91 \pm 5.06$ & $72.30 \pm 13.76$ & $-18.62 \pm 15.71$ & -23.93 to -13.30 & $<0.0001$ \\
\hline $\begin{array}{l}\text { Monocyte } \\
\text { count (\%) }\end{array}$ & 36 & $4.46 \pm 2.12$ & $6.16 \pm 3.33$ & $1.70 \pm 4.64$ & 0.13 to 3.26 & 0.035 \\
\hline
\end{tabular}

$\S$. Paired-samples t-test. $P<0.05$ is significant.

In comparison to controls, patients with GD after treatment with carbimazole had significantly higher monocyte counts $(p=0.035)$.
On the other hand, TLR4 expression on monocyte was not significantly different between both groups (Table 3).

Table 3. Comparison of TLR4 on monocytes and monocyte count in GD cases after treatment versus controls

\begin{tabular}{|c|c|c|c|c|c|}
\hline \multirow[t]{2}{*}{ Variable } & $\begin{array}{c}\text { GD cases after } \\
\text { treatment } \\
(n=36)\end{array}$ & $\begin{array}{c}\text { Controls } \\
(n=36)\end{array}$ & & & \\
\hline & Mean $\pm S D$ & Mean $\pm S D$ & Difference & $95 \% \mathrm{Cl}$ & $\begin{array}{c}P- \\
\text { value }^{\S}\end{array}$ \\
\hline TLR4 on monocytes (\%) & $72.30 \pm 13.76$ & $66.30 \pm 27.24$ & -6.00 & -16.14 to 4.15 & NS \\
\hline Monocyte count (\%) & $6.16 \pm 3.33$ & $3.88 \pm 2.13$ & -2.27 & -3.59 to -0.96 & 0.001 \\
\hline
\end{tabular}

$\S$. Independent-samples t-test. . $P>0.05$ is no significant (NS).

As shown in Table 4 and Figure 3, TLR4 expression on monocytes showed a good diagnostic value for discrimination between GD patients at baseline and controls with high 
degrees of sensitivity and specificity. On the other hand, monocyte count did not show a good diagnostic value for discrimination between the two groups.

Table 4. Receiver-operating characteristics (ROC) curve analysis for discrimination between GD cases before treatment and controls using TLR4 on monocytes or monocyte count

\begin{tabular}{lcc} 
& \multicolumn{2}{c}{ Marker } \\
\hline ROC parameter & TLR4 on monocytes & Monocyte count \\
\hline Area under the ROC curve(AUC) & 0.879 & 0.613 \\
\hline Significance level P (Area=0.5) & $<0.0001$ & 0.100 \\
\hline Sensitivity & 86.1 & 72.2 \\
\hline Specificity & 80.6 & 61.1 \\
\hline+ PV & 81.6 & 65 \\
\hline -PV & 85.3 & 68.7 \\
\hline
\end{tabular}

$+P V=$ positive predictive value, $-\mathrm{PV}=$ negative predictive value.

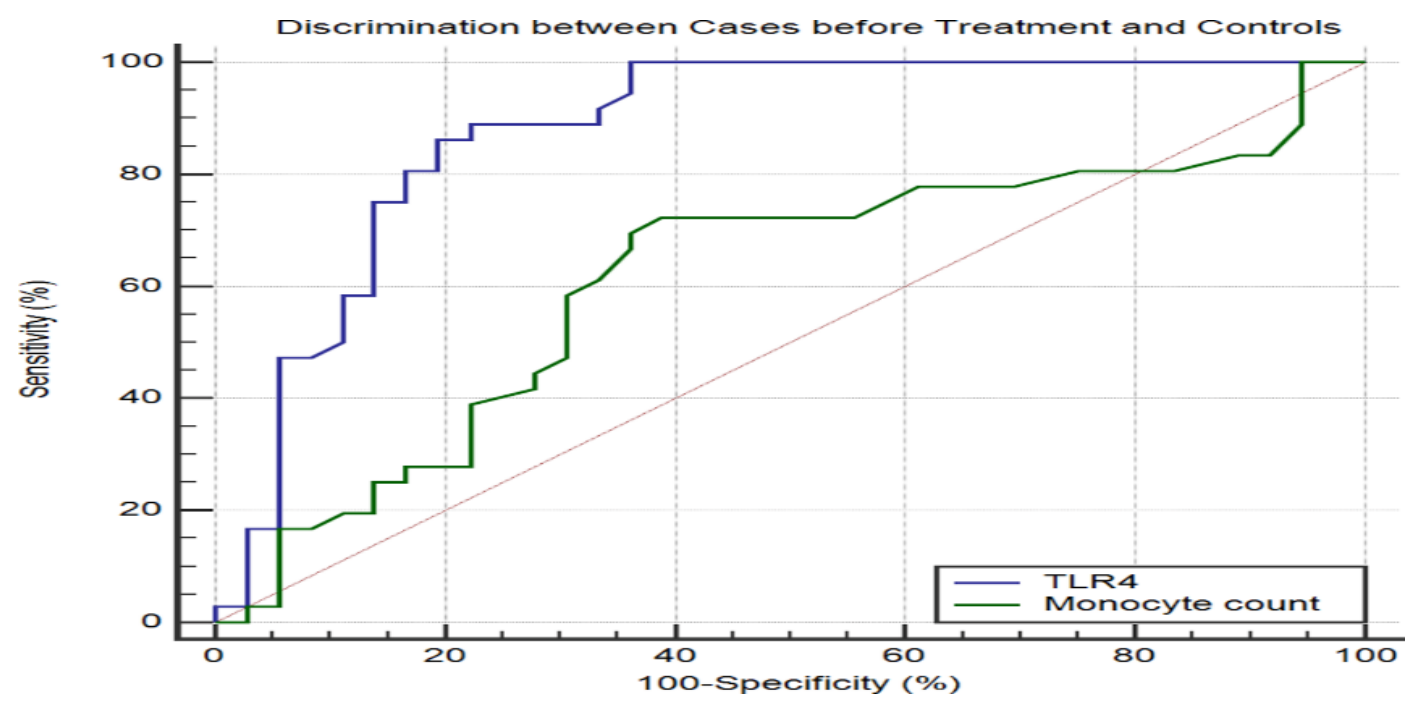

Figure 3. Receiver-operating characteristics (ROC) curves for discrimination between GD cases before treatment and controls using TLR4 or monocyte count.

TLR4 expression on monocytes was significantly higher among GD patients with exophthalmos than among dose without exophthalmos ( $p$ value< 0.0001 ) (Table 5 ), and significantly higher among those with moderate to severe exophthalmos than among those with mild exophthalmos $(P<0.001)$ (Table 6).

Table 5. Comparison of TLR4 on monocytes in GD cases with or without exophthalmos.

\begin{tabular}{lccccc}
\hline & $\begin{array}{c}\text { GD cases without } \\
\text { exophthalmos } \\
(n=16)\end{array}$ & $\begin{array}{c}\text { GD cases with } \\
\text { exophthalmos } \\
(n=20)\end{array}$ & & & \\
\hline Variable & Mean $\pm S D$ & Mean $\pm S D$ & Difference & $95 \% \mathrm{Cl}$ & $P$-value \\
\hline TLR4 on monocytes (\%) & $92.51 \pm 3.81$ & $89.64 \pm 5.64$ & 2.87 & -0.49 to 6.22 & $<0.0001$ \\
\hline
\end{tabular}

$\S$. Independent-samples t-test. . $P<0.05$ is significant. 
Table 6. Comparison of TLR4 on monocytes in GD cases with mild or moderate to severe exophthalmos.

\begin{tabular}{lccccc}
\hline & $\begin{array}{c}\text { GD cases with } \\
\text { mild } \\
\text { exophthalmos } \\
(n=9)\end{array}$ & $\begin{array}{c}\text { GD cases with } \\
\text { moderate to } \\
\text { severe } \\
\text { exophthalmos } \\
(n=11)\end{array}$ & & & \\
Variable & Mean \pm SD & Mean \pm SD & Difference & $95 \% \mathrm{Cl}$ & $P$-value \\
\hline TLR4 on monocytes (\%) & $84.89 \pm 3.62$ & $93.53 \pm 3.60$ & -8.64 & -12.05 to - & $<0.001$ \\
\hline
\end{tabular}

$\S$ I Independent-samples t-test. . $P<0.05$ is significant.

\section{Discussion}

The aim of the current study was to measure Toll like receptor 4 (TLR4) expression on peripheral blood mononuclear cells in patients with GD before and after 6 months or more of therapy with Carbimazole in comparison to a group of normal controls. Data of the present study results indicated significant differences in expression of TLR4 in GD patients before treatment, after achieving euthyroid state and in the control group $(P<0.0001)$.

In the literature, there are few data on the role of TLR in GD. Cho et al., 2017 observed significant differences in genotypic frequencies of TLR4 polymorphisms in patients with GD. They demonstrated that GD patients showed higher frequencies of the TLR4 rs1927911 C allele than controls, among a group of Korean children with differences of those with autoimmune thyroid disease (AITD) (44 with HD and 60 with GD). ${ }^{9}$ Peng et al., 2018 demonstrated a significantly increased expression of TLRs in the peripheral blood of patients with newly diagnosed GD and patients with Hashimoto thyroiditis as compared to a group of controls. ${ }^{10}$ Polak et al., 2019 reported significantly increased expression of TLR4 on T and B lymphocytes among GD patients than among controls. In addition, TLR4 decreased among those patients after achieving euthyrodism but remained significantly higher than the control group. ${ }^{11}$

Conversely, Peng et al., 2016 demonstrated an equivalent expression of TLR1, 4, 5, 6, 7, and 8 in AITD patients and controls. On the other hand, the expression of TLR2, TLR3, TLR9, and
TLR10 were significantly increased in both patients with Hashimoto thyroiditis (HT) and those with GD subjects than in controls, but no difference was found between HT and GD patients. ${ }^{12}$ Similarly, Xiao et al., 2014 studied the association between genetic polymorphisms of TLR4 and TLR5 genes and GD in a sample of Chinese Cantonese population. ${ }^{13}$ They demonstrated that TLR5 polymorphisms were significantly associated with GD. However, there was no significant association between TLR4 polymorphism and GD. Another study, Liao et al., $2010^{14}$ observed lack of association of TLR4 polymorphisms with GD.

To the best of our knowledge, no previous study demonstrated the diagnostic value of TLR4 for discrimination between GD cases at baseline and controls. The current study showed a good diagnostic value of TLR4 expression on monocytes for discrimination between the two groups with a sensitivity of $86.1 \%$ and specificity of $80.6 \%$.

Our study showed that TLR4 expression on monocytes was significantly higher in GD patients with exophthalmos in comparison to patients without exophthalmos ( $p<0.0001$ ). Furthermore, TLR4 showed higher expression on monocytes in GD patients with moderate to severe exophthalmos than in those with mild exophthalmos $(P<0.001)$. Polak et al., 2019 in their study reported increased expression of TLR4 on $T$ and B lymphocytes among GD patients who had more severe clinical manifestations. ${ }^{11}$

Conversely, Cho et al., 2017 found lower frequency of the TLR4 in thyroid associated ophthalmopathy group, whereas a non-thyroid 
associated ophthalmopathy group showed a higher frequency of TLR4 than the control group. ${ }^{9}$ In the present study, however, we observed a significant increase in monocytic count among GD patients after treatment in comparison to GD patients at baseline. This was similar to a study conducted by Szydełko et al., 2020 who showed that patients with thyroid axis dysfunctions had significantly lower monocytic counts compared to those with compensated thyroid function values. ${ }^{15}$

Conversely, Turan, 2019 found that monocyte counts were significantly higher in untreated GD patients than in euthyroid GD patients after anti-thyroid treatment and in the controls. ${ }^{16}$

In conclusion, our study showed statistically significant differences in the expression of TLR4 on monocytes in GD patients before receiving antithyroid therapy, after achieving euthyrodism, and in control subjects. The mean percentage of TLR4 expression on monocytic cells in GD patients was higher than in the control group, which may suggest an important role in mechanisms of non-specific and specific immunity in the pathogenesis of the Graves disease.

\section{Author Contributions}

S.S.H. was responsible for conception and design of the study, data analysis, formal analysis and review corresponding. M.N.F was responsible for data curation, formal analysis, manuscript editing, manuscript preparation and review corresponding. D.S.S was responsible for data analysis, methodology, review corresponding and manuscript revision. H.M.E was responsible for review corresponding and manuscript revision. D.A.M was responsible for review corresponding and manuscript revision and Y.G.M who performed design of the study, literature search, data acquisition and analysis, methodology, manuscript preparation and writing the original draft. All authors read and approved the final manuscript.

\section{Declaration of Conflicting Interests}

The author(s) declared no potential conflicts of interest with respect to the research, authorship, and/or publication of this article.

\section{Funding}

The author(s) denies receipt of any financial support for the research, authorship, and/or publication of this article.

\section{Ethical approval}

The study protocol was reviewed and approved by the Ethical Committee of the Faculty of Medicine, Ain Shams University (Approval No. FMASU MD 265/2018).

\section{Informed consent}

A written informed consent was obtained from each participant included in our study.

\section{References}

1. Antonelli, A., Ferrari, SM., Corrado, A., et al. (2015). Autoimmune thyroid disorders. Autoimmune Reviews. doi: 10.1016/j.autrev.2014.10.016. Epub 2014 Oct 25. PMID: 25461470.

2. Terry, J., and Laszlo, H. (2016). Graves Disease. The new England journal of medicine; 375:15521565. DOI: 10.1056/NEJMra1510030

3. Kawashima, A., Tanigawa, K., Akama, T., et al. (2011). Innate immune activation and thyroid autoimmunity. The Journal of Clinical Endocrinology \& Metabolism, Volume 96, Issue 12, Pages 3661-3671

4. El-Zayat, S., Sibaii, H., Mannaa, F. (2019). Toll-like receptors activation, signaling, and targeting: an overview. Bull Natl Res Cent 43, 187.

5. Miyake, K. (2004). Endotoxin recognition molecules, Toll-like receptor 4-MD-2. Seminars in Immunology; 16:11-16

6. Kawashima, A., Yamazaki, K., Hara, T., et al. (2013). "Demonstration of innate immune responses in the thyroid gland: potential to sense danger and a possible trigger for autoimmune reactions." Thyroid: official journal of the American Thyroid Association vol. 23,4: 477-87. doi:10.1089/thy.2011.0480

7. Ross, DS., Burch, HB., Cooper, DS., et al. (2016). American Thyroid Association Guidelines for Diagnosis and Management of Hyperthyroidism and Other Causes of Thyrotoxicosis. Oct;26(10):1343-1421. doi: 10.1089/thy.2016.0229. Erratum in: Thyroid. 2017 Nov; 27(11):1462. PMID: 27521067.

8. Bartalena, L., Baldeschi, L., Dickinson, A., et al. (2008). "Consensus statement of the European Group on Graves' orbitopathy (EUGOGO) on 
management of GO," European Journal of Endocrinology, vol. 158, no. 3, pp. 273-285, 158(3):273-85

9. Cho, W., Jang, J., Choi, E., et al. (2017). "Association of Polymorphisms in Toll-Like Receptors 4 and 9 with Autoimmune Thyroid Disease in Korean Pediatric Patients", International Journal of Endocrinology, vol. 2017, Article ID 2304218

10. Peng, S., Sun, X., Wang, X., et al. (2018). Myeloid related proteins are up-regulated in autoimmune thyroid diseases and activate toll-like receptor 4 and pro-inflammatory cytokines in vitro. International Immunopharmacology doi: 10.1016/j.intimp.2018.04.009. PMID: 29656212.

11. Polak, A., Grywalska, E., Klatka, J., et al. (2019). Toll-Like Receptors-2 and -4 in Graves' DiseaseKey Players or Bystanders? International Journal of Molecular Sciences. 20(19):4732.

12. Peng, S., Li, C., Wang, X., et al. (2016). "Increased Toll-Like Receptors Activity and TLR Ligands in Patients with Autoimmune Thyroid Diseases." Frontiers in immunology vol. 7 578. 9 doi:10.3389/fimmu.2016.00578
13. Xiao, W., Liu, Z., Lin, J., et al. (2014). Association of TLR4 and TLR5 gene polymorphisms with Graves' disease in Chinese Cantonese population. Human Immunology. Jul;75(7):609-13. doi: 10.1016/j.humimm.2014.05.001. Epub 2014 May 14. PMID: 24830583

14. Liao, W., Chen, R., Lin, H., et al. (2010). Toll-like receptor gene polymorphisms are associated with susceptibility to graves' ophthalmopathy in Taiwan males. BMC medical genetics, 154. doi:10.1186/1471-2350-11-154

15. Szydełko, J., Litwińczuk, M., Szydełko, M., et al. (2020). Neutrophil-to-Lymphocyte, Monocyte-toLymphocyte and Platelet-to-Lymphocyte Ratios in Relation to Clinical Parameters and Smoking Status in Patients with Graves' Orbitopathy-Novel Insight into Old Tests. Journal of Clinical Medicine. doi: 10.3390/jcm9103111. PMID: 32993174; PMCID: PMC7600876.

16. Turan, E. (2019). Evaluation of neutrophil-tolymphocyte ratio and hematologic parameters in patients with Graves' disease. Bratislava medical journal. doi: 10.4149/BLL_2019_076. PMID: 31223030. 